\title{
On the creation of conjugate points for Hamiltonian systems
}

\author{
G Contreras $\dagger \|$, R Iturriagał and H Sánchez-Morgado $\S$ \\ † Depto. de Matemática. PUC-Rio, R Marquês de São Vicente 225, 22453-900 Rio de Janeiro, \\ Brazil \\ $\ddagger$ CIMAT, AP 402, 3600, Guanajuato Gto, Mexico \\ $\S$ Instituto de Matemáticas, UNAM Ciudad Universitaria CP 04510, Cd de Mexico, Mexico
}

Received 15 May 1997, in final form 7 November 1997

Recommended by V Lazutkin

\begin{abstract}
For a fixed Hamiltonian $H$ on the cotangent bundle of a compact manifold $M$ and a fixed energy level $e$, we prove that the set $\mathcal{A}_{e}$ of potentials $\phi$ on $M$ such that the Hamiltonian flow of $H+\phi$ is Anosov, is the interior in the $C^{2}$ topology of the set $\mathcal{B}_{e}$ of potentials such that the flow has no conjugate points.
\end{abstract}

AMS classification scheme numbers: $58 \mathrm{~F} 05,58 \mathrm{~F} 15$

\section{Introduction}

Let $M$ be a closed connected Riemannian manifold and $T^{*} M$ its cotangent bundle. By a Hamiltonian on $T^{*} M$ we shall mean a $C^{\infty}$ function $H: T^{*} M \rightarrow \mathbb{R}$ satisfying the following conditions.

(a) Convexity: for all $q \in M, p \in T_{q}^{*} M$, the Hessian matrix $\left(\partial^{2} H / \partial p_{i} \partial p_{j}\right)(q, p)$ (calculated with respect to linear coordinates on $T_{q}^{*} M$ ) is positive definite.

(b) Superlinearity:

$$
\lim _{|p| \rightarrow \infty} \frac{H(q, p)}{|p|}=+\infty
$$

The Hamiltonian equation for $H$ is defined as

$$
q^{\prime}=H_{p} \quad p^{\prime}=-H_{q}
$$

where $H_{q}$ and $H_{p}$ are the partial derivatives with respect to $q$ and $p$. Observe that the Hamiltonian function $H$ is constant along the solutions of (1). Its level sets $\Sigma_{e}=H^{-1}(e)$ are called energy levels of $H$. Then the compactness of $M$ and the superlinearity hypothesis imply that the energy levels are compact. Since the Hamiltonian vector field (1) is Lipschitz, the solutions of (1) are defined on all $\mathbb{R}$. Denote by $\psi_{t}$ the corresponding Hamiltonian flow on $T^{*} M$.

Let $\pi: T^{*} M \rightarrow M$ be the canonical projection and define the vertical subspace on $\theta \in T^{*} M$ by $\mathbb{V}(\theta)=\operatorname{ker}(\mathrm{d} \pi(\theta))$. Two points $\theta_{1}, \theta_{2} \in T^{*} M$ are said to be conjugate if $\theta_{2}=\psi_{\tau}\left(\theta_{1}\right)$ for some $\tau \neq 0$ and $\mathrm{d} \psi_{\tau}\left(\mathbb{V}\left(\theta_{1}\right)\right) \cap \mathbb{V}\left(\theta_{2}\right) \neq\{0\}$.

|| Current address: CIMAT, AP 402, 3600, Guanajuato Gto, Mexico. 
We recall that a smooth flow $\psi_{t}$ generated by the vector field $X$ on a compact manifold $N$, is called Anosov if there exist continuous invariant sub-bundles $E^{u}, E^{s}$ of $T N$ and constants $c>0, a>0$, such that

(a) $T N=E^{s} \oplus E^{u} \oplus \mathbb{R} X$

(b) $\left|\mathrm{d} \psi_{t}\left(v^{s}\right)\right| \leqslant c e^{-a t}\left|v^{s}\right|$ for all $t>0, v^{s} \in E^{s}$ $\left|\mathrm{d} \psi_{t}\left(v^{u}\right)\right| \leqslant c e^{a t}\left|v^{u}\right|$ for all $t<0, v^{u} \in E^{u}$.

For $e \in \mathbb{R}$, let $\mathcal{A}_{e}$ be the set of $\phi \in C^{\infty}(M)$ such that the flow of $H+\phi$ is Anosov in $(H+\phi)^{-1}(e)$ and let $\mathcal{B}_{e}$ be the set of $\phi \in C^{\infty}(M)$ such that $(H+\phi)^{-1}(e)$ contains no conjugate points. As is well known $\mathcal{A}_{e}$ is open in $C^{k}$ topology and $\mathcal{B}_{e}$ is closed. On the other hand Paternain and Paternain [Pa] have shown that $\mathcal{A}_{e}$ is contained in $\mathcal{B}_{e}$. They also proved that if the flow is Anosov on the energy level $\Sigma_{e}$, then $e>\max \{H(q, 0): q \in M\}$ and $\Sigma_{e}$ is diffeomorphic to the unitary sphere bundle in $T^{*} M$. In the appendix of [An] Margulis proved that if a compact 3-manifold admits an Anosov flow, then its fundamental group has exponential growth. Therefore there is no Hamiltonian on $T^{*} T^{2}$ with Anosov energy levels. On the other hand the geodesic flow for the plane metric on $T^{2}$ has no conjugate points anywhere. Of course there is plenty of Hamiltonians with Anosov energy levels if $M$ has a metric with negative curvature. The purpose of this paper is to prove the following

Theorem 1. The interior of $\mathcal{B}_{e}$ in the $C^{2}$ topology is $\mathcal{A}_{e}$.

This theorem is an extension to the Hamiltonian setting of a result of Ruggiero [Ru] for the geodesic flow. Our main tools are the Green bundles, theorem 2 below describing $\mathcal{A}_{e}$ inside $\mathcal{B}_{e}$, and the index form in the Lagrangian setting.

\section{Green bundles and hyperbolicity}

The Green bundles were constructed for the geodesic flow by Green in [Gr] using the Jacobi equation. For Hamiltonian systems they were constructed in [C-I], where proofs for the statements in this section can be found.

Suppose that the orbit of $\theta \in T^{*} M$ does not contain conjugate points and $H(\theta)=e$ is a regular value of $H$. Then there exist two $\mathrm{d} \psi_{t}$-invariant Lagrangian sub-bundles $\mathbb{E}$, $\mathbb{F} \subset T\left(T^{*} M\right)$ along the orbit of $\theta$ given by

$$
\begin{aligned}
& \mathbb{E}(\theta)=\lim _{t \rightarrow+\infty} \mathrm{d} \psi_{-t}\left(\mathbb{V}\left(\psi_{t}(\theta)\right)\right) \\
& \mathbb{F}(\theta)=\lim _{t \rightarrow+\infty} \mathrm{d} \psi_{t}\left(\mathbb{V}\left(\psi_{-t}(\theta)\right)\right) .
\end{aligned}
$$

Moreover, $\mathbb{E}(\theta) \cup \mathbb{F}(\theta) \subset T_{\theta} \Sigma, \mathbb{E}(\theta) \cap \mathbb{V}(\theta)=\mathbb{F}(\theta) \cap \mathbb{V}(\theta)=\{0\}, X(\theta) \in \mathbb{E}(\theta) \cap \mathbb{F}(\theta)$ and $\operatorname{dim} \mathbb{E}(\theta)=\operatorname{dim} \mathbb{F}(\theta)=\operatorname{dim} M$, where $X(\theta)=\left(H_{p},-H_{q}\right)$ is the Hamiltonian vector field and $\Sigma=H^{-1}\{e\}$.

Fix a Riemannian metric on $M$ and the corresponding induced metric on $T^{*} M$. Then $T_{\theta} T^{*} M$ splits as a direct sum of two Lagrangian subspaces: the vertical subspace $\mathbb{V}(\theta)=\operatorname{ker}(\mathrm{d} \pi(\theta))$ and the horizontal subspace $\mathbb{H}(\theta)$ given by the kernel of the connection map. Using the isomorphism $K: T_{\theta} T^{*} M \rightarrow T_{\pi(\theta)} M \times T_{\pi(\theta)}^{*} M, \xi \mapsto\left(\mathrm{d} \pi(\theta) \xi, \nabla_{\pi \xi} \theta\right)$, we can identify $\mathbb{H}(\theta) \approx T_{\pi(\theta)} M \times\{0\}$ and $\mathbb{V}(\theta) \approx\{0\} \times T_{\pi(\theta)}^{*} M \approx T_{\pi(\theta)} M$. Let $E \subset T_{\theta} T^{*} M$ be an $n$-dimensional subspace such that $E \cap \mathbb{V}(\theta)=\{0\}$. Then $E$ is the graph of some linear map $S: \mathbb{H}(\theta) \rightarrow \mathbb{V}(\theta)$. It can be verified that $E$ is Lagrangian if and only if in symplectic coordinates $S$ is symmetric. 
Take $\theta \in T^{*} M$ and $\xi \in T_{\theta} T^{*} M=\mathbb{H}(\theta) \oplus \mathbb{V}(\theta) \approx T_{\pi(\theta)} M \oplus T_{\pi(\theta)} M$. Writing $\mathrm{d} \psi_{t}(\xi)=(h(t), v(t))$, we obtain the Hamiltonian Jacobi equations

$$
\dot{h}=H_{p q} h+H_{p p} v \quad \dot{v}=-H_{q q} h-H_{q p} v
$$

where the covariant derivatives are evaluated along $\pi\left(\psi_{t}(\theta)\right)$, and $H_{q q}, H_{q p}, H_{p p}$ and $H_{q q}$ are linear operators on $T_{\pi(\theta)} M$, that in local coordinates coincide with the matrices of partial derivatives $\left(\partial^{2} H / \partial q_{i} \partial q_{j}\right),\left(\partial^{2} H / \partial q_{i} \partial p_{j}\right),\left(\partial^{2} H / \partial p_{i} \partial p_{j}\right)$ and $\left(\partial^{2} H / \partial p_{i} \partial q_{j}\right)$. Moreover, since the Hamiltonian $H$ is convex, then $H_{p p}$ is positive definite.

Let $E$ be a Lagrangian subspace of $T_{\theta} T^{*} M$. Suppose that for $t$ in some interval ] $-\varepsilon, \varepsilon[$ we have that $\mathrm{d} \psi_{t}(E) \cap \mathbb{V}\left(\psi_{t}(\theta)\right)=\{0\}$. Then we can write $\mathrm{d} \psi_{t}(E)=$ graph $S(t)$, where $S(t): \mathbb{H}\left(\psi_{t} \theta\right) \rightarrow \mathbb{V}\left(\psi_{t} \theta\right)$ is a symmetric map. That is, if $\xi \in E$ then

$$
\mathrm{d} \psi_{t}(\xi)=(h(t), S(t) h(t)) .
$$

Using equation 2 we have that

$$
\dot{S} h+S\left(H_{p q} h+H_{p p} S h\right)=-H_{q q} h-H_{q p} S h .
$$

Since this holds for all $h \in \mathbb{H}\left(\psi_{t}(\theta)\right)$ we obtain the Hamiltonian version of the Riccati equation, which is well known in the Lagrangian setting (e.g. [Ge])

$$
\dot{S}+S H_{p p} S+S H_{p q}+H_{q p} S+H_{q q}=0 .
$$

Suppose there are no conjugate points in the interval $[-T, T]$, we can construct matrix solutions $\left(Z_{T}(t), V_{T}(t)\right),\left(Z_{-T}(-t), V_{-T}(-t)\right), 0 \leqslant t \leqslant T$ to the Jacobi equation with $Z_{ \pm T}(0)=I, Z_{T}(T)=Z_{-T}(-T)=0$. Let $K_{ \pm T}(t)=V_{ \pm T}(t) Z_{ \pm T}(t)^{-1}$ be the corresponding solutions to the Riccati equation. Let $E_{T}(\theta)=\mathrm{d} \psi_{-T}\left(\mathbb{V}\left(\psi_{T}(\theta)\right)\right)$ and $F_{T}(\theta)=\mathrm{d} \psi_{T}\left(\mathbb{V}\left(\psi_{-T}(\theta)\right)\right)$, then $\mathrm{d} \psi_{t}\left(E_{T}(\theta)\right)=\operatorname{graph} K_{T}(t)$ and $\mathrm{d} \psi_{t}\left(F_{T}(\theta)\right)=$ graph $K_{-T}(t)$. If there are no conjugate points along all the orbit we have that $K_{T}(0) \rightarrow$ $\boldsymbol{S}(\theta)$, and $K_{-T}(0) \rightarrow \boldsymbol{U}(\theta)$ as $T \rightarrow+\infty$; where $\boldsymbol{S}(\theta), \boldsymbol{U}(\theta)$ are the the symmetric solutions of the Riccati equation (3) corresponding to the Green bundles $\mathbb{E}(\theta)=\operatorname{graph}(\boldsymbol{S}(\theta)$ ) and $\mathbb{F}(\theta)=\operatorname{graph}(\boldsymbol{U}(\theta))$.

The following theorem relates the hyperbolicity of the flow $\psi_{t}$ with the transversality of the Green bundles.

Theorem 2. Let $\Sigma=H^{-1}\{e\}$ be a regular energy level without conjugate points. Then the following statements are equivalent:

(a) $\left.\psi_{t}\right|_{\Sigma}$ is Anosov,

(b) for all $\theta \in \Sigma, \mathbb{E}(\theta)$ and $\mathbb{F}(\theta)$ are transversal in $T_{\theta} \Sigma$,

(c) for all $\theta \in \Sigma, \mathbb{E}(\theta) \cap \mathbb{F}(\theta)=\mathbb{R} X(\theta)$,

(d) if $\theta \in \Sigma, v \in T_{\theta} \Sigma, v \notin \mathbb{R} X(\theta)$ then $\sup _{t \in \mathbb{R}}\left|\mathrm{d} \psi_{t}(\theta) \cdot v\right|=+\infty$.

\section{The index form}

The index form is more naturally defined in the Lagrangian setting. Since the Hamiltonian is convex in the fibres we can use the Legendre transform to obtain a Lagrangian $L: T M \rightarrow \mathbb{R}$ (see $[\mathrm{Ar}])$. The Euler-Lagrange equations

$$
\frac{\mathrm{d}}{\mathrm{d} t} x=v \quad \frac{\mathrm{d}}{\mathrm{d} t} L_{v}=L_{x}
$$

are equivalent to the Hamilton equations (1). We derive the Jacobi equation in this Lagrangian setting. Let $\gamma(t)$ be a solution of the Euler-Lagrange equation. Considering a 
variation $f(s, t)$ of $\gamma(t)=f(0, t)$ made of solutions $t \mapsto f(s, t)$ of the Euler-Lagrange equation and taking the covariant derivative $\frac{\mathrm{D}}{\mathrm{d} s}$, we obtain the Jacobi equation

$$
\frac{\mathrm{D}}{\mathrm{d} t}\left(L_{v x} k+L_{v v} \dot{k}\right)=L_{x x} k+L_{x v} \dot{k}
$$

where $k=\frac{\partial f}{\partial s}(0, t), \dot{k}=\frac{\mathrm{D}}{\mathrm{d} t} \frac{\partial f}{\partial s}$ and the derivatives of $L$ are evaluated on $\gamma(t)=f(0, t)$. Here we have used that $\frac{\mathrm{D}}{\mathrm{d} s} \frac{\partial F}{\partial t}=\frac{\mathrm{D}}{\mathrm{d} t} \frac{\partial F}{\partial s}$ for the variation map $F(s, t)=L_{v}\left(f(s, t), \frac{\partial f}{\partial s}(s, t)\right) \in$ $T^{*} M$, where $\frac{\mathrm{D}}{\mathrm{d} s}$ and $\frac{\mathrm{D}}{\mathrm{d} t}$ are the covariant derivatives on the Riemannian manifold $T^{*} M$. The linear operators $L_{x x}, L_{x v}, L_{v v}$ coincide with the corresponding matrices of partial derivatives in local coordinates. The solutions of (5) satisfy

$$
\mathrm{D} \varphi_{t}(k(0), \dot{k}(0))=(k(t), \dot{k}(t)) \in T_{\gamma(t)}(T M)
$$

where $\varphi_{t}$ is the Lagrangian flow on $T M$.

Let $\Omega_{T}$ be the set of continuous piecewise $C^{2}$ vector fields along $\gamma_{[-T, T]}$. Define the index form on $\Omega_{T}$ by

$$
I(\xi, \eta)=\int_{-T}^{T}\left(\dot{\xi} L_{v v} \dot{\eta}+\dot{\xi} L_{v x} \eta+\xi L_{x v} \dot{\eta}+\xi L_{x x} \eta\right) \mathrm{d} t
$$

which is the second variation of the action functional for variations $f(s, t)$ with $\frac{\partial f}{\partial s} \in \Omega_{T}$.

The following expresion of the index form is taken from [C-I] and originally due to Clebsch [Cl]. Let $\theta \in T^{*} M$ and suppose that the orbit of $\theta, \psi_{t}(\theta),-T \leqslant t \leqslant T$ does not have conjugate points. Let $Z(t), V(t)$ be a matrix solution of the Hamiltonian Jacobi equation (2) such that $\operatorname{det} Z(t) \neq 0$. Let $\eta \in \Omega_{T}$ and define $\zeta \in \Omega_{T}$ by $\eta(t)=Z(t) \zeta(t)$. Then the integrand of $I(\eta, \eta)$ in (6) is $(Z \dot{\zeta})^{*}\left(H_{p p}\right)^{-1} Z \dot{\zeta}+\left[(Z \zeta)^{*} V \zeta\right]^{\prime}$. Since $\eta$ and $\zeta$ are continuous, we have that

$$
I(\eta, \eta)=\int_{-T}^{T}(Z \dot{\zeta})^{*}\left(H_{p p}\right)^{-1} Z \dot{\zeta} \mathrm{d} t+\left.(Z \zeta)^{*} V \zeta\right|_{-T} ^{T} .
$$

Corollary 1. If $\theta \in T^{*} M$ and the segment $\left\{\psi_{t}(\theta) \mid t \in[-T, T]\right\}$ has no conjugate points then the index form is positive definite on

$\Gamma_{T}=\left\{\xi:[-T, T] \rightarrow T M \mid \xi(t) \in T_{\pi \psi_{t} \theta} M, \xi\right.$ is piecewise $\left.C^{2}, \xi(-T)=0, \xi(T)=0\right\}$.

Proof. Let $\xi \in \Gamma_{T}, \xi \neq 0$. Write $\xi(t)=Z(t) \zeta(t)$. Since $\operatorname{det} Z(t) \neq 0$ for all $t \in[-T, T]$, $\zeta(-T)=0, \zeta(T)=0$ and $\zeta \neq 0$, then $\dot{\zeta} \neq 0$. Now use formula (7).

One can extend formula (7) using different solutions $\left(Z_{i}, V_{i}\right)$ of the Jacobi equation on disjoint subintervals \{]$t_{i}, t_{i+1}[: i=0, \ldots, N\}$ of $[-T, T]$. Then

$$
I(\eta, \eta)=\sum_{i=0}^{N} \int_{t_{i}}^{t_{i+1}}\left(Z_{i} \dot{\zeta}_{i}\right)^{*}\left(H_{p p}\right)^{-1} Z_{i} \dot{\zeta}_{i} \mathrm{~d} t+\left[\left(Z_{i} \zeta_{i}\right)^{*} V_{i} \zeta_{i}\right]_{t_{i}^{+}}^{t_{i+1}^{-}}
$$

where $\left.\eta\right|_{t_{i}, t_{i+1}[}=Z_{i} \zeta_{i}$

\section{Proof of theorem 1}

The proof will proceed as follows. We take a system that has no conjugate points but is not Anosov, make a small perturbation in a neighbourhood of an orbit segment to end up with a system that has conjugate points. We will prove that the perturbed system has conjugate points by showing that the index form is not positive definite on that segment. 
Let $\varphi \in \mathcal{B}_{e} \backslash \mathcal{A}_{e}$ and $\varepsilon>0$. We can assume that $\varphi=0$. By theorem 2 there are $\theta \in \Sigma$, $v \in T_{\theta} \Sigma$ such that $v \in \mathbb{E}(\theta) \cap \mathbb{F}(\theta) \backslash \mathbb{R} X(\theta)$. Let $\gamma(t)=\pi \psi_{t}(\theta)$. Since $X(\theta) \in \mathbb{E}(\theta) \cap \mathbb{F}(\theta)$ and $\dot{\gamma}(0)=\mathrm{d} \pi(X(\theta)) \neq 0$, we can assume that $|\mathrm{d} \pi(v)|=1$ and $\langle\mathrm{d} \pi(v), \dot{\gamma}(0)\rangle=0$. Let $C>0$ be such that the segment $\left.\gamma\right|_{[-C, C]}$ is injective, and let $T>C$.

Let $Z_{ \pm T}(t), V_{ \pm T}(t), K_{ \pm T}(t), 0 \leqslant t \leqslant T$ and $E_{T}(\theta), F_{T}(\theta)$ be as in section 2 .

Let $\zeta_{T} \in E_{T}(\theta), \eta_{T} \in F_{T}(\theta)$ be such that $\mathrm{d} \pi \zeta_{T}=\mathrm{d} \pi \eta_{T}=\mathrm{d} \pi v$.

Define $\xi_{T}:[-T, T] \rightarrow T M$ by

$$
\xi_{T}(t)= \begin{cases}\mathrm{d} \pi \mathrm{d} \psi_{t}\left(\eta_{T}\right)=Z_{-T}(t) \mathrm{d} \pi(v) & \text { for } t \in[-T, 0] \\ \mathrm{d} \pi \mathrm{d} \psi_{t}\left(\zeta_{T}\right)=Z_{T}(t) \mathrm{d} \pi(v) & \text { for } t \in[0, T] .\end{cases}
$$

By (8)

$$
\begin{aligned}
I\left(\xi_{T}, \xi_{T}\right)= & \xi_{T}(T)^{*} K_{T}(T) \xi_{T}(T)-\xi_{T}(-T)^{*} K_{-T}(-T) \xi_{T}(-T) \\
& +\xi_{T}(0)^{*}\left(K_{-T}(0)-K_{T}(0)\right) \xi_{T}(0) \\
= & \mathrm{d} \pi(v)^{*}\left(K_{-T}(0)-K_{T}(0)\right) \mathrm{d} \pi(v) .
\end{aligned}
$$

There exist $\lambda \in(0, C / 2)$ and $T_{0}>0$ such that $\left|\bar{\xi}_{T}(t)\right|^{2}>\frac{1}{2}$ for $|t| \leqslant \lambda$ and $T>T_{0}$, where $\bar{\xi}_{T}(t)$ is the component of $\xi(t)$ orthogonal to $\dot{\gamma}(t)$.

Since $K_{T}(0) \rightarrow \boldsymbol{S}(\theta), K_{-T}(0) \rightarrow \boldsymbol{U}(\theta)$ as $T \rightarrow \infty$ and $v=(\mathrm{d} \pi(v), \boldsymbol{S}(\theta) \mathrm{d} \pi(v))=$ $(\mathrm{d} \pi(v), \boldsymbol{U}(\theta) \mathrm{d} \pi(v))$, we have that $\left|\left(K_{-T}(0)-K_{T}(0)\right) d \pi(v)\right| \rightarrow 0$ as $T \rightarrow \infty$. Fix $T$ sufficiently large to have $I\left(\xi_{T}, \xi_{T}\right)<\varepsilon \lambda / 4$. Henceforth we omit the subscript $T$ in $\xi$.

Choose coordinates $\bar{x}=\left(x_{1}, \ldots, x_{n}\right): U \rightarrow[-C, C] \times \mathbb{R}^{n-1}$ on a tubular neighbourhood of $\gamma([-C, C])$ such that $\bar{x} \circ \gamma([-C, C])=[-C, C] \times\{\mathbf{0}\}$ and $\left\{\frac{\partial}{\partial x_{1}}, \ldots, \frac{\partial}{\partial x_{n}}\right\}$ is an orthogonal frame over the points of $\gamma([-C, C])$.

Define $\tilde{H}=H+\phi$ with $\phi\left(x_{1}, \ldots, x_{n}\right)=\frac{1}{2} f\left(x_{1} / C\right) f(|x| / \delta) \varepsilon|x|^{2}$, where $\boldsymbol{x}=$ $\left(x_{2}, x_{3}, \ldots, x_{n}\right)$ and $f: \mathbb{R} \rightarrow \mathbb{R}$ is a $C^{\infty}$ bump function with support in $[-1,1]$ which is 1 on $\left[-\frac{1}{2}, \frac{1}{2}\right]$. Thus $\tilde{H}_{p}=H_{p}, \tilde{L}=L-\phi, \tilde{L}_{v}=L_{v}, \tilde{L}_{v v}=L_{v v}, \tilde{L}_{v x}=L_{v x}$. Since

$\phi_{x}=\frac{1}{2} \varepsilon\left(C^{-1} f^{\prime}\left(x_{1} / C\right) f(|\boldsymbol{x}| / \delta)|\boldsymbol{x}|^{2}, f\left(x_{1} / C\right)\left[f^{\prime}(|\boldsymbol{x}| / \delta)|\boldsymbol{x}| / \delta+2 f(|\boldsymbol{x}| / \delta)\right] \boldsymbol{x}\right)$

and $\phi_{x x}=\frac{1}{2} \varepsilon\left[\begin{array}{cc}a(\bar{x}) & \boldsymbol{b}(\bar{x}) \\ \boldsymbol{b}(\bar{x})^{t} & \boldsymbol{A}(\bar{x})\end{array}\right]$ where

$a=C^{-2} f^{\prime \prime}\left(x_{1} / C\right) f(|x| / \delta)|x|^{2} \quad \boldsymbol{b}=C^{-1} f^{\prime}\left(x_{1} / C\right)\left[f^{\prime}(|x| / \delta)|x| / \delta+2 f(|x| / \delta)\right] \boldsymbol{x}$

$\boldsymbol{A}=f\left(\frac{x_{1}}{C}\right)\left(\left[f^{\prime \prime}(|\boldsymbol{x}| / \delta) / \delta^{2}+(3 / \delta|\boldsymbol{x}|) f^{\prime}(|\boldsymbol{x}| / \delta)\right] \boldsymbol{x} \otimes \boldsymbol{x}+\left[f^{\prime}(|\boldsymbol{x}| / \delta)|\boldsymbol{x}| / \delta+2 f(|\boldsymbol{x}| / \delta)\right] I\right)$ we have $\left|\phi_{x}\right| \leqslant c \delta \varepsilon,\left|\phi_{x x}\right| \leqslant c \varepsilon$ and then

$$
|\tilde{X}(\theta)-X(\theta)| \leqslant c \delta \varepsilon \quad|D \tilde{X}(\theta)-D X(\theta)| \leqslant c \varepsilon
$$

where $c$ is a constant. Using (6), we have

$$
\begin{aligned}
\tilde{I}(\xi, \xi)=I(\xi, \xi) & +\int_{-T}^{T}\left(\dot{\xi}\left(L_{v v}\left(\tilde{\psi}_{t}(\theta)\right)-L_{v v}\left(\psi_{t}(\theta)\right)\right) \dot{\xi}+2 \dot{\xi}\left(L_{v x}\left(\tilde{\psi}_{t}(\theta)\right)-L_{v x}\left(\psi_{t}(\theta)\right)\right) \xi\right) \mathrm{d} t \\
& +\int_{-T}^{T} \xi\left(\tilde{L}_{x x}\left(\tilde{\psi}_{t}(\theta)\right)-\tilde{L}_{x x}\left(\psi_{t}(\theta)\right)+\tilde{L}_{x x}\left(\psi_{t}(\theta)\right)-L_{x x}\left(\psi_{t}(\theta)\right)\right) \xi \mathrm{d} t .
\end{aligned}
$$

Since $\lim _{\delta \rightarrow 0} \tilde{\psi}_{t}(\theta)=\psi_{t}(\theta)$ uniformly on $t \in[-T, T]$, for $\delta>0$ sufficiently small we have

$\tilde{I}(\xi, \xi) \leqslant I(\xi, \xi)+\int_{-T}^{T} \xi\left(\tilde{L}_{x x}\left(\psi_{t}(\theta)\right)-L_{x x}\left(\psi_{t}(\theta)\right)\right) \xi \mathrm{d} t+\varepsilon \lambda / 4$. 
Let $\Delta(t)=\xi(t)\left(\tilde{L}_{x x}\left(\psi_{t}(\theta)\right)-L_{x x}\left(\psi_{t}(\theta)\right)\right) \xi(t)$. If $\gamma(t) \in \bar{x}^{-1}([-C, C] \times\{\boldsymbol{0}\})$,

$$
\Delta(t)=-\xi(t) \phi_{x x}(\gamma(t)) \xi(t)=-\varepsilon \xi(t)\left[\begin{array}{cc}
0 & \mathbf{0} \\
\mathbf{0} & f\left(x_{1} \circ \gamma(t) / C\right)
\end{array}\right] \xi(t) .
$$

Then

$$
\Delta(t)= \begin{cases}-\varepsilon f\left(x_{1} \circ \gamma(t) / C\right)|\bar{\xi}|^{2} & \text { if } \gamma(t) \in \bar{x}^{-1}([-C, C] \times\{\mathbf{0}\}) \\ 0 & \text { if } \gamma(t) \notin B(C, \delta)\end{cases}
$$

where $B(C, \delta)=\left\{q \in M: \bar{x}(q) \in[-C, C] \times B_{\delta}(\mathbf{0})\right\}$. Therefore

$$
\int_{-T}^{T} \Delta(t) \mathrm{d} t<-\varepsilon \lambda+\int_{D(T, C, \delta)} \Delta(t) \mathrm{d} t
$$

where $D(T, C, \delta)=([-T, T] \backslash[-C, C]) \cap \gamma^{-1}(B(C, \delta))$.

Lemma 1. If the orbit $\gamma(t)$ is not periodic

$$
\lim _{\delta \rightarrow 0} \int_{D(T, C, \delta)} \Delta(t) \mathrm{d} t=0
$$

Proof. If the segment $\left.\gamma\right|_{[-T, T]}$ is injective, then we can choose $\delta>0$ sufficiently small to have $D(T, C, \delta)=\emptyset$ and hence the integral vanishes.

Suppose that $([-T, T] \backslash[-C, C]) \cap \gamma^{-1}(\gamma([-C, C]))=\left\{s_{1}, \ldots, s_{N}\right\}$. Let $t_{1}, \ldots, t_{N} \in$ $[-C, C]$ with $\gamma\left(s_{i}\right)=\gamma\left(t_{i}\right)$. Let $\bar{\gamma}_{i}^{\prime}$ be the component of $(\bar{x} \circ \gamma)^{\prime}\left(s_{i}\right)$ orthogonal to $(\bar{x} \circ \gamma)^{\prime}\left(t_{i}\right)$. Let $r>0$ be such that $\left|\bar{\gamma}_{i}^{\prime}\right|>2 r$ for $i=1, \ldots, N$ and define $h_{i}(t)=$ $\left\langle\bar{x} \circ \gamma(t), \bar{\gamma}_{i}^{\prime}\right\rangle /\left|\bar{\gamma}_{i}^{\prime}\right|$ for $\left|t-s_{i}\right|$ small. Then $\exists \alpha>0$ such that $h_{i}^{\prime}(t)>r$ and $\left|h_{i}(t)\right|>\left|t-s_{i}\right| r$ for $\left|t-s_{i}\right|<\alpha$. If $\delta$ is sufficiently small, then $\gamma(t) \notin B(C, \delta)$ if $\delta / r<\left|t-s_{i}\right|$ for any $i=1, \ldots, N$ and so

$$
\int_{D(T, C, \delta)}|\Delta(t)| \mathrm{d} t \leqslant \sum_{i=1}^{N} \int_{s_{i}-(\delta / r)}^{s_{i}+(\delta / r)}|\Delta(t)| \mathrm{d} t .
$$

The lemma follows.

Lemma 2. If the orbit $\gamma(t)$ is periodic, then

$$
\lim _{\delta \rightarrow 0} \int_{D(T, C, \delta)} \Delta(t) \mathrm{d} t \leqslant 0 .
$$

Proof. Let $\tau$ be the period of $\gamma$. Defining

$$
D^{\prime}(T, C, \delta)=\left([-T, T] \backslash \bigcup_{m=0}^{\infty}[m \tau-C, m \tau+C]\right) \cap \gamma^{-1}(B(C, \delta))
$$

for $k=[T+C / \tau]$ we have

$$
\int_{D(T, C, \delta)} \Delta(t) \mathrm{d} t=\int_{D^{\prime}(T, C, \delta)} \Delta(t) \mathrm{d} t+\sum_{m=1}^{k} \int_{m \tau-C}^{m \tau+C} \Delta(t) \mathrm{d} t .
$$

As in lemma 1 one proves that $\lim _{\delta \rightarrow 0} \int_{D^{\prime}(T, C, \delta)} \Delta(t) \mathrm{d} t=0$.

By (13) we have $\sum_{m=1}^{k} \int_{m \tau-C}^{m \tau+C} \Delta(t) \mathrm{d} t \leqslant 0$.

Thus, for $\delta>0$ sufficiently small we have $\tilde{I}(\xi, \xi) \leqslant I(\xi, \xi)-\varepsilon \lambda+\varepsilon \lambda / 4<0$ and corollary 1 implies that $H+\phi$ has conjugate points in $(H+\phi)^{-1}(e)$. 


\section{Acknowledgments}

GC was partially supported by CNPq-Brazil. RI was partially supported by Conacyt-Mexico, grant 0324P-E. H SM was partially supported by PAPIIT, grant IN-103795.

\section{References}

[An] Anosov D V and Sinai Ya G 1967 Some smooth ergodic systems Russ. Math. Surv. 22 103-68

[Ar] Arnold V I 1989 Mathematical Methods of Classical Mechanics (New York: Springer)

[Cl] Clebsch A 1958 Über die reduktion der zweiten variation auf ihre einfachste form J. Reine Angew. Math. 55 254-76

[C-I] Contreras G and Iturriaga R 1998 Convex Hamiltonians without conjugate points Ergod. Theor. Dynam. Syst. to appear. Preprint available at http://www.ma.utexas.edu/mp_arc

[Ge] Gelfand I M and Fomin S V 1963 Calculus of Variations (Englewood Cliffs, NJ: Prentice-Hall)

[Gr] Green L W 1958 A theorem of E Hopf Michigan Math. J. 5 31-4

[Pa] Paternain G and Paternain M 1994 On Anosov energy levels of convex Hamiltonian systems Math. Z. 217 367-76

[Ru] Ruggiero R O 1991 On the creation of conjugate points Math. Z. 208 41-55 